\title{
A NEW SPECIAL FORM OF THE LINEAR ELEMENT OF A SURFACE
}

\author{
BY \\ JESSE DOUGLAS
}

1. Introduction and statement of results. The great circles of a sphere form a family of $\infty^{2}$ curves having the following three important properties:

(1) They are geodesics of the sphere.

(2) They are a linear system; that is, a point transformation exists which converts them into the straight lines of a plane. Indeed, central projection of the sphere on any plane not passing through its center will accomplish such a transformation. An equivalent statement is that it is possible to introduce coordinates $u, v$ on the spherical surface so that the totality of great circles is represented by the general linear equation: $a u+b v+c=0$.

(3) The angular excess of any triangle $A B C$ formed by great circles is proportional to the area of the triangle:

$$
\mathcal{E}=A+B+C-\pi=k \mathcal{A},
$$

where the factor of proportionality $k$ is equal to the Gaussian curvature of the sphere: $k=1 / R^{2}$.

It is evident that all the geometric entities and properties involved in these three statements are invariant under any bending or isometric transformation of the spherical surface $\left({ }^{1}\right)$ together with its great circles; this means a point transformation into a family of $\infty^{2}$ curves upon another surface so that $d s=d s^{\prime}$, where $d s$ denotes the element of length of the sphere and $d s^{\prime}$ the corresponding element of the transformed surface. According to a fundamental theorem of Gauss, the Gaussian curvature $K$ of the transformed surface must be the same as that of the sphere, therefore constant. Evidently, too, the geodesics of the sphere go over into the geodesics of the transformed surface. It follows that the three stated properties are possessed also by the geodesics of any surface of constant Gaussian curvature.

Let us denote by $(\mathcal{F}, S)$ the geometric configuration consisting of a family $\mathcal{F}$ of $\infty^{2}$ curves upon a surface $S$. Then it is obvious that, in respect of the possession or non-possession of any of our three properties, the configuration $(\mathcal{F}, S)$ is completely equivalent to any configuration $\left(\mathcal{F}^{\prime}, S^{\prime}\right)$ derived therefrom by isometric transformation. Any two such isometric configurations will

Presented to the Society, April 27, 1940; received by the editors March 5, 1940. This paper was received by the editors of the Annals of Mathematics, May 11, 1939, accepted by them, and later transferred to these Transactions.

(1) Meaning a properly limited region of the spherical surface. As is well known, the sphere as a whole is indeformable. In general, all our considerations will be local or differentialgeometric. 
therefore be regarded as essentially identical. In other words, all that is relevant concerning the surface $S$ is its first fundamental form

$$
d s^{2}=E d u^{2}+2 F d u d v+G d v^{2} .
$$

The family $\mathcal{F}$ may always be defined by a differential equation of second order:

$$
v^{\prime \prime}=\phi\left(u, v, v^{\prime}\right) \quad\left(v^{\prime}=d v / d u, v^{\prime \prime}=d^{2} v / d u^{2}\right) .
$$

Thus any configuration $(\mathcal{F}, S)$ is represented analytically by a system of functions $\left[E(u, v), F(u, v), G(u, v), \phi\left(u, v, v^{\prime}\right)\right]$.

With every two properties that may be selected from the three stated at the beginning, we may associate a corresponding converse problem. Thus we may ask for all configurations $(\mathcal{F}, S)$ which have:

(a) properties (1) and (3),

(b) properties (1) and (2),

(c) properties (2) and (3).

The answer to the converse question (a) is classical. According to a theorem of Gauss, the angular excess $\mathcal{E}$ of any triangle $A B C$ formed by three geodesics of a surface $S$ is given by the formula $\left({ }^{2}\right)$

$$
\varepsilon=\iint K d \omega
$$

over $A B C$,

where $d \omega$ denotes the element of area. By the law of the mean, this gives $\mathcal{E}=K(m) \mathcal{A}$, where $K(m)$ denotes the value of the Gaussian curvature at some point $m$ of $A B C$, while $A$ denotes the area of this triangle. It follows immediately that, as the triangle $A B C$ shrinks to any fixed point $p$ of $S$,

$$
\lim \varepsilon / \mathcal{A}=K(p) .
$$

Property (3) then implies that the Gaussian curvature of the surface $S$ is constant: $K(p)=k$ for every point $p$ of $S$. That the family $\mathcal{F}$ consists of the geodesics of $S$ is part of the data of problem (a).

The answer to the converse question (b) is also classical, having been given by Beltrami in $1865\left(^{3}\right)$. He proved the theorem: if a surface $S$ can be represented point by point on a plane so that the geodesics of $S$ correspond to the straight lines of the plane, then $S$ has constant Gaussian curvature. Thus, again, the only solution of the converse problem is the one which is known $a$ priori.

The converse problem (c). It is curious that the converse problem (c) has not hitherto been studied. Here I have found the solution $(\mathcal{F}, S)$ to be more general than the geodesics of a surface of constant curvature. In fact, the

(2) In formula (2.2) of the next section, let $1 / \rho=0$, expressing the geodesic character of the sides of the triangle.

$\left({ }^{3}\right)$ E. Beltrami, Opere Matematiche, vol. 1, pp. 262-280. 
complete solution is given by the formulas which follow, whose derivation constitutes the purpose of the present paper.

In formulating our results, it is convenient to use-instead of general coordinates $u, v$ upon the surface $S$, wherein $d s^{2}$ has the form (1.2)-minimal coordinates, wherein

$$
d s^{2}=2 F d u d v .
$$

The characteristic property of minimal coordinates is that the coordinate lines $u=$ const., $v=$ const. have zero length $\left({ }^{4}\right)$, or are the minimal lines of the surface $S$. Such coordinates are determined only up to an arbitrary transformation $u_{1}=\phi(u), v_{1}=\psi(v)$, which preserves the minimal character of the coordinate lines.

Let $U_{1}, U_{2}$ denote any two functions of $u$ alone, and $V_{1}, V_{2}$ any two functions of $v$ alone. Form the determinants

$$
\mathrm{I}=\left|\begin{array}{cc}
U_{1}+V_{1} & U_{1}^{\prime} \\
U_{2}+V_{2} & U_{2}^{\prime}
\end{array}\right|, \quad \mathrm{II}=\left|\begin{array}{cc}
U_{1}+V_{1} & V_{1}^{\prime} \\
U_{2}+V_{2} & V_{2}^{\prime}
\end{array}\right|,
$$

where the accent denotes differentiation. We always suppose $U_{1}, U_{2}, V_{1}, V_{2}$ so chosen that $I \neq 0, I I \neq 0$. Then we shall prove that the most general configuration $(\mathcal{F}, S)$ having the properties (2), (3) is represented by the formulas:

$$
\mathcal{F}: \quad v^{\prime \prime}=B v^{\prime}+C v^{\prime 2}
$$

where

$$
\left(1.6^{\prime}\right) \quad B=\frac{\partial}{\partial u}(\log \mathrm{I}-2 \log \mathrm{II}), \quad C=\frac{\partial}{\partial v}(2 \log \mathrm{I}-\log \mathrm{II}),
$$

while, for the surface $S$,

$$
S: \quad 2 k F=\frac{\partial^{2}}{\partial u \partial v}(\log \mathrm{I}+\log \mathrm{II}) .
$$

With the help of a simple determinant transformation $\left({ }^{5}\right)$, we find

$$
\frac{\partial^{2}}{\partial u \partial v} \log \mathrm{I}=\frac{\mathrm{II}}{\mathrm{I}^{2}} \cdot\left|\begin{array}{cc}
U_{1}^{\prime} & U_{1}^{\prime \prime} \\
U_{2}^{\prime} & U_{2}^{\prime \prime}
\end{array}\right|, \quad \frac{\partial^{2}}{\partial u \partial v} \log \mathrm{II}=\frac{\mathrm{I}}{\mathrm{II}^{2}} \cdot\left|\begin{array}{cc}
V_{1}^{\prime} & V_{1}^{\prime \prime} \\
V_{2}^{\prime} & V_{2}^{\prime \prime}
\end{array}\right|
$$

hence the expanded form of (1.7) is

$$
2 k F=\frac{\mathrm{II}}{\mathrm{I}^{2}} \cdot\left|\begin{array}{cc}
U_{1}^{\prime} & U_{1}^{\prime \prime} \\
U_{2}^{\prime} & U_{2}^{\prime \prime}
\end{array}\right|+\frac{\mathrm{I}}{\mathrm{II}^{2}} \cdot\left|\begin{array}{cc}
V_{1}^{\prime} & V_{1}^{\prime \prime} \\
V_{2}^{\prime} & V_{2}^{\prime \prime}
\end{array}\right| .
$$

The case $k=0$. In interpreting these results, it is important to distinguish

(4) Of course, these coordinate lines must be imaginary.

(5) Formula (3.19). 
the case $k=0$ from $k \neq 0$. If $k=0$, property (3) becomes the statement that the sum of the angles of every triangle of $\mathcal{F}$ is two right angles. In addition, $\mathcal{F}$ is required to be linear, by property (2). Now, an arbitrary surface $S$ is capable of conformal representation upon a plane $P$, and in infinitely many ways, since we may combine any given conformal representation of $S$ on $P$ with an arbitrary conformal transformation of $P$ into itself: $u^{\prime}+i v^{\prime}=f(u+i v)$. Let $\mathcal{F}$ denote the family of $\infty^{2}$ curves on $S$ which results from the family of all straight lines of the plane $P$ by any conformal map of $S$ on $P$. Then $\mathcal{F}$ is obviously linear, and also the angle-sum of every triangle of $\mathcal{F}$ is two right angles, since these are conformally invariant properties which belong to the straight lines. Thus, an arbitrary surface $S$ carries infinitely many families of curves $\mathcal{F}$ which are linear and in which every triangle has an angle-sum of two right angles.

This finds expression in the formula $\left(1.7^{\prime}\right)$ by the circumstance that when $k=0$ this formula implies no restriction on the function $F$, that is, on the $d s^{2}$ of $S$, but rather only a condition on the functions $U_{1}, U_{2}, V_{1}, V_{2}$ which determine the family $\mathcal{F}$. Indeed, if $k=0$, it follows from (1.7) that

$$
\mathrm{I} \cdot \mathrm{II}=U_{3} V_{3},
$$

and from $\left(1.7^{\prime}\right)$ that either

$$
\frac{\mathrm{I}}{\mathrm{II}}=\frac{U_{4}}{V_{4}}
$$

where

$$
U_{4}=\left|\begin{array}{ll}
U_{1}^{\prime} & U_{1}^{\prime \prime} \\
U_{2}^{\prime} & U_{2}^{\prime \prime}
\end{array}\right|^{1 / 3}, \quad V_{4}=-\left|\begin{array}{cc}
V_{1}^{\prime} & V_{1}^{\prime \prime} \\
V_{2}^{\prime} & V_{2}^{\prime \prime}
\end{array}\right|^{1 / 3},
$$

or else that

$$
\left|\begin{array}{cc}
U_{1}^{\prime} & U_{1}^{\prime \prime} \\
U_{2}^{\prime} & U_{2}^{\prime \prime}
\end{array}\right|=0, \quad\left|\begin{array}{ll}
V_{1}^{\prime} & V_{1}^{\prime \prime} \\
V_{2}^{\prime} & V_{2}^{\prime \prime}
\end{array}\right|=0 .
$$

By (1.9) and $\left(1.9^{\prime}\right)$,

$$
\mathrm{I}=U_{5} V_{5}, \quad \mathrm{II}=U_{6} V_{6}
$$

therefore

$$
\frac{\partial^{2}}{\partial u \partial v} \log \mathrm{I}=0, \quad \frac{\partial^{2}}{\partial u \partial v} \log \mathrm{II}=0,
$$

whence by (1.8), since by hypothesis $\mathrm{I} \neq 0, \mathrm{II} \neq 0$, we deduce

$$
\left|\begin{array}{cc}
U_{1}^{\prime} & U_{1}^{\prime \prime} \\
U_{2}^{\prime} & U_{2}^{\prime \prime}
\end{array}\right|=0, \quad\left|\begin{array}{cc}
V_{1}^{\prime} & V_{1}^{\prime \prime} \\
V_{2}^{\prime} & V_{2}^{\prime \prime}
\end{array}\right|=0 .
$$


(1.10) is the same as (1.12), which therefore subsists in either case. (1.12) implies the existence of linear relations with constant coefficients:

$$
c_{1} U_{1}+c_{2} U_{2}=c_{3}, \quad c_{1}^{\prime} V_{1}+c_{2}^{\prime} V_{2}=c_{3}^{\prime},
$$

where either $c_{1}$ or $c_{2} \neq 0$ and either $c_{1}^{\prime}$ or $c_{2}^{\prime} \neq 0$.

It is evident by the defining formulas (1.5) that, under the conditions (1.13), the functions I, II must have the forms (1.11). Therefore, by (1.6'),

$$
B=U, \quad C=V \text {; }
$$

accordingly, the differential equations (1.6) of $\mathcal{F}$ have the form

$$
v^{\prime \prime}=U v^{\prime}+V v^{\prime 2} \text {. }
$$

It is easily verified that this is the general form of differential equation for a family $\mathcal{F}$ derivable by conformal transformation: $u_{1}=\phi(u), v_{1}=\psi(v)$, from the straight lines of a plane: $v_{1}^{\prime \prime}=0$-explicitly, $U=\phi^{\prime \prime}(u) / \phi^{\prime}(u)$, $V=-\psi^{\prime \prime}(v) / \psi^{\prime}(v)$. In summary, we have a proof of the following theorem:

If a family $\mathcal{F}$ of $\infty^{2}$ curves on a surface $S$ is linear, and the sum of the angles in every triangle of $\mathcal{F}$ is two right angles, then $\mathcal{F}$ must be a conformal image of the $\infty^{2}$ straight lines of a plane.

In a previous paper $\left(^{(}\right)$, the author proved this theorem synthetically. The first statement and proof is an analytic one by E. Kasner $\left({ }^{7}\right)$.

The case $k \neq 0$. Of more interest is the case $k \neq 0$. Then formula (1.7) or $\left(1.7^{\prime}\right)$ really specializes the surface $S$ : its $d s^{2}$ must have, in minimal coordinates, the form

$$
\begin{aligned}
d s^{2} & =\frac{1}{k} \frac{\partial^{2}}{\partial u \partial v}(\log \mathrm{I}+\log \mathrm{II}) d u d v \\
& =\frac{1}{k}\left\{\frac{\mathrm{II}}{\mathrm{I}^{2}} \cdot\left|\begin{array}{cc}
U_{1}^{\prime} & U_{1}^{\prime \prime} \\
U_{2}^{\prime} & U_{2}^{\prime \prime}
\end{array}\right|+\frac{\mathrm{I}}{\mathrm{II}^{2}} \cdot\left|\begin{array}{cc}
V_{1}^{\prime} & V_{1}^{\prime \prime} \\
V_{2}^{\prime} & V_{2}^{\prime \prime}
\end{array}\right|\right\} d u d v .
\end{aligned}
$$

Upon all and only such surfaces $S$ can curve families $\mathcal{F}$ be found with properties (2) and (3).

In order that this form of the surface $S$ shall not be degenerate, it is necessary and sufficient (besides $\mathrm{I} \neq 0, \mathrm{II} \neq 0$ ) that $F \neq 0$, or

$$
\frac{\partial^{2}}{\partial u \partial v}(\log \mathrm{I}+\log \mathrm{II}) \neq 0 \text {. }
$$

But in the contrary case, we have seen by the calculations (1.9)-(1.13) that we must have (1.12) or its equivalent (1.13). Conversely, it is evident that (1.12) implies $F=0$.

( ${ }^{\circ}$ Number 2 of the list of references at the end.

( $\left.{ }^{7}\right)$ Reference [1]. 
Hence, under the hypothesis $I \neq 0, I I \neq 0$, the formula (1.15) will give a nondegenerate surface $S$ when and only when linear relations of the form (1.13) do not subsist simultaneously between $U_{1}, U_{2}$ and between $V_{1}, V_{2}$. If $U_{1}, U_{2}$ are not both constant and $V_{1}, V_{2}$ are not both constant, the condition that relations of the form (1.13) or (1.12) shall not hold simultaneously is sufficient to guarantee in addition that $\mathrm{I} \neq 0, \mathrm{II} \neq 0$.

This completes our description of the special form of the linear element of the surface $S$ signified by the title of the present paper.

An indication that this type of surface $S$ is more general than one of constant curvature results by a count of arbitrary functions. The most general form of the $d s^{2}$ of a surface of constant curvature $c$ referred to minimal coordinates is $\left({ }^{8}\right)$

$$
d s^{2}=\frac{4 U^{\prime} V^{\prime}}{c(U-V)^{2}} d u d v,
$$

thus involving, besides the arbitrary constant $c$, only the two arbitrary functions $U$ of $u$ and $V$ of $v$, which determine the distribution of parametric values $u, v$ over the two systems of minimal lines respectively. The formula (1.15), on the other hand, involves four general functions $U_{1}, U_{2}, V_{1}, V_{2}$, subject only to the slight restrictions of linear independence which we have mentioned. Of these four functions, two correspond to an arbitrary transformation $u_{1}=\phi(u)$, $v_{1}=\psi(v)$ on the surface $S$ which conserves the minimal lines, so that only two of the arbitrary functions are really effective in varying the form of $S$. We may say that, if isometric surfaces are regarded as identical, there are only $\infty^{1}$ surfaces of constant curvature, depending on the value $c$ of this curvature, whereas the category of surfaces $S$ with properties (2) and (3) involves two arbitrary functions of one variable.

This indication is, of course, not completely decisive, since there remains the question of whether $U_{1}, U_{2}, V_{1}, V_{2}$ are all essential. To obtain a definite proof that the formula $\left(1.7^{\prime}\right)$ contains surfaces not of constant curvature, we may calculate the Gaussian curvature by the formula

$$
K=-\frac{1}{F} \frac{\partial^{2}}{\partial u \partial v} \log F=\frac{F_{u} F_{v}-F F_{u v}}{F^{3}} .
$$

The result is a rational function of $U_{1}, U_{2}, V_{1}, V_{2}$ and their derivatives of the first three orders. A partial calculation suffices to show that this rational function does not reduce identically to a constant when all the quantities mentioned are considered as independent variables - as they may be, since the functions $U_{1}, U_{2}, V_{1}, V_{2}$ are arbitrary, and they and any finite number of their derivatives are therefore capable of taking any assigned values for any finite number of given values of $(u, v)$. Consequently, we can arrange to give

(8) Cf. G. Darboux, Théorie Générale des Surfaces, 1887 edition, vol. 1, p. 30. Write $x=U$ $y=V$ in formula (1), p. 30 . 
these functions and those of their derivatives which appear in the expression for $K$ such particular values at any two chosen points $\left(u_{1}, v_{1}\right),\left(u_{2}, v_{2}\right)$ that $K\left(u_{1}, v_{1}\right) \neq K\left(u_{2}, v_{2}\right)$; therefore $K$ will not be a constant.

2. Conditions for the property $\varepsilon=k \mathcal{A}$. We begin the proof of the results stated in $\$ 1$ by recalling the formula of Gauss-Bonnet $\left({ }^{9}\right)$. If $\Gamma$ denote any closed curve with continuously turning tangent, bounding a region $R$, then

$$
\int_{\Gamma} \frac{d s}{\rho}+\iint_{R} K d \omega=2 \pi
$$

where $1 / \rho$ is the geodesic curvature of $\Gamma$. In case the curve $\Gamma$ has corners at the points $P_{i}(i=1,2, \cdots, m)$, then this formula must be modified as follows:

$$
\int_{\Gamma} \frac{d s}{\rho}+\iint_{R} K d \omega+\sum_{i=1}^{m} \theta_{i}=2 \pi .
$$

Here $\theta_{i}$ represents the angle, taken with proper sign, between the sensed tangents to the two arcs of $\Gamma$ which form the corner at $P_{i}$.

Let us apply formula (2.1) to any triangle $A B C$ formed by three curves of $\mathcal{F}$. The boundary $\Gamma$ of this triangle has corners at $P_{1}, P_{\mathrm{s}}, P_{3}=A, B, C$, and $\theta_{1}=\pi-A, \theta_{2}=\pi-B, \theta_{3}=\pi-C$, where $A, B, C$ denote the interior angles of the triangle. Consequently, by substitution in (2.1),

$$
\int_{\Gamma} \frac{d s}{\rho}+\iint_{A B C} K d \omega=A+B+C-\pi=\varepsilon .
$$

By property (3),

$$
\varepsilon=k \mathcal{A}=\iint_{A B C} k d \omega
$$

therefore

$$
\int_{\Gamma} \frac{d s}{\rho}=\iint_{A B C}(k-K) d \omega=\iint_{A B C}(k-K) W d u d v
$$

since

$$
d \omega=W d u d v
$$

where $W=(E G-F)^{1 / 2}$.

Every polygon whose sides are curves of $\mathcal{F}$ can be decomposed into triangles of $\mathcal{F}$. It follows, by the additive nature of both contour and surface integration, that the formula (2.4) applies to any polygon of $\mathcal{F}$; that is, if $\Gamma$ denotes the boundary and $R$ the interior of any polygon of $F$, we have

$$
\int_{\Gamma} \frac{d s}{\rho}=\iint_{R}(k-K) W d u d v .
$$

${ }^{(9)}$ See W. Blaschke, Vorlesungen über Differentialgeometrie, 1921, p. 108. 
By Green's theorem, the surface integral over $R$ can be expressed as a contour integral over $\Gamma$ :

$$
\iint_{R}(k-K) W d u d v=\int_{\Gamma} P_{1} d u+Q_{1} d v
$$

where $P_{1}, Q_{1}$ are any two functions of $u, v$ which obey

$$
\frac{\partial Q_{1}}{\partial u}-\frac{\partial P_{1}}{\partial v}=(k-K) W
$$

- the existence of such functions is obvious.

Relations (2.6) and (2.7) give

$$
\int_{\Gamma}\left(\frac{d s}{\rho}-P_{1} d u-Q_{1} d v\right)=0
$$

for every polygon $\Gamma$ of $F$. This implies that the same integral taken over any polygonal path between any two fixed points of $S$ does not depend on the path itself but only on its end-points. (By a "polygonal path" we mean one composed of a finite number of arcs of curves of $F$.) According to a standard theorem $\left({ }^{10}\right)$, it follows that the element of integration in (2.9) is an exact differential:

$$
\frac{d s}{\rho}-P_{1} d u-Q_{1} d v=\lambda_{u} d u+\lambda_{v} d v
$$

where the subscripts denote partial differentiation of the arbitrary function $\lambda(u, v)$. Thus we have

$$
\frac{d s}{\rho}=P d u+Q d v,
$$

where $P=P_{1}+\lambda_{u}, Q=Q_{1}+\lambda_{v}$. Obviously, $P, Q$ also obey the condition (2.8):

$$
Q_{u}-P_{v}=(k-K) W,
$$

since $\lambda_{u v}$ cancels in the process of substitution.

Conversely, it is easily seen that if (2.10) is obeyed along every curve of a family $\mathcal{F}$, and $P, Q$ are related by (2.11), then $\mathcal{F}$ has property (3), as expressed by (2.3).

Formula (2.10) by itself defines a type of curve family called a velocity family(11). Thus property (3) is characteristic of a particular kind of velocity family-one where formula (2.11) is obeyed.

$\left.{ }^{(10}\right)$ It is easily seen to be sufficient for the application of this theorem that the condition of independence of the path of integration apply merely to polygonal paths of $\mathcal{F}$.

(11) The name is due to E. Kasner, these Transactions, vol. 10 (1909), p. 213. The geodesics of a Weyl metric are a general velocity family; see H. Weyl, Raum, Zeit, Materie, 3d edition, 
In minimal coordinates, where $d s^{2}=2 F d u d v$, the geodesic curvature $1 / \rho$ of any curve $v=v(u)$ is given by $\left({ }^{12}\right)$

$$
\frac{d s}{\rho}=\frac{-v^{\prime \prime}+\left(F_{u} / F\right) v^{\prime}-\left(F_{v} / F\right) v^{\prime 2}}{2 i v^{\prime}} d u .
$$

Therefore, for a velocity family, we have by (2.10):

$$
v^{\prime \prime}=B v^{\prime}+C v^{\prime 2},
$$

where

$$
\begin{aligned}
& B=F_{u} / F-2 i P=(\log F)_{u}-2 i P, \\
& C=-F_{v} / F-2 i Q=-(\log F)_{v}-2 i Q .
\end{aligned}
$$

Conversely, a family $\mathcal{F}$ whose differential equation in minimal coordinates is of the type (2.13), where $B, C$ are $a n y$ functions of $u, v$, obeys (2.10) with $P, Q$ defined by (2.14). That is: the form (2.13) is characteristic of velocity families.

Let us now apply the condition (2.11) by calculating

$$
C_{u}-B_{v}=-2(\log F)_{u v}-2 i\left(Q_{u}-P_{v}\right) .
$$

In minimal coordinates, where $E=0, G=0, W=\left(E G-F^{2}\right)^{1 / 2}=i F$, the Gaussian curvature $K$ is expressed by (1.17), so that the condition (2.11) becomes

$$
Q_{u}-P_{v}=i k F+i(\log F)_{u v} .
$$

This gives, when substituted in (2.15),

$$
C_{u}-B_{v}=2 k F \text {. }
$$

Conversely, (2.17) gives (2.16) when substituted in the identity (2.15).

In summary: property (3) is expressed in minimal coordinates by the formulas (2.13), (2.17).

3. Linearity of the family $\mathcal{F}$. We now have to impose the additional property (2) of linearity on the family $\mathcal{F}$.

If we apply an arbitrary coordinate transformation

$$
u_{1}=\phi(u, v), \quad v_{1}=\psi\left(u, v^{\prime}\right)
$$

to $\mathcal{F}$, the effect on the derivatives $v^{\prime}, v^{\prime \prime}$ is as follows:

$$
v_{1}^{\prime}=\frac{\psi_{u}+\psi_{v} v^{\prime}}{\phi_{u}+\phi_{v} v^{\prime}}
$$

1919, p. 112. Cf. also C. H. Rowe [4]. The term "zyklisches Netz" used by J. Radon, following Blaschke, denotes the same thing as a velocity family; see J. Radon [3].

(12) Blaschke, loc. cit., p. 117. Write $E=0, G=0, u^{\prime}=1, u^{\prime \prime}=0$. 


$$
\begin{aligned}
v_{1}^{\prime \prime}= & \left\{\left(\phi_{u}+\phi_{v} v^{\prime}\right)\left(\psi_{u u}+2 \psi_{u v} v^{\prime}+\psi_{v v} v^{\prime 2}+\psi_{v} v^{\prime \prime}\right)\right. \\
& \left.-\left(\psi_{u}+\psi_{v} v^{\prime}\right)\left(\phi_{u u}+2 \phi_{u v} v^{\prime}+\phi_{v v} v^{\prime 2}+\phi_{v} v^{\prime \prime}\right)\right\} /\left(\phi_{u}+\phi_{v} v^{\prime}\right)^{3} .
\end{aligned}
$$

Suppose that after this transformation the finite equation of $\mathcal{F}$ has the linear form $v_{1}=a u_{1}+b$, or the differential equation of $\mathcal{F}$ becomes $v_{1}^{\prime \prime}=0$. Then in the original coordinate system $(u, v)$ the differential equation of $\mathcal{F}$ is, by (3.3),

$$
v^{\prime \prime}=A+B v^{\prime}+C v^{\prime 2}+D v^{\prime 3}
$$

where

$$
\begin{aligned}
& A=\frac{\psi_{u} \phi_{u u}-\phi_{u} \psi_{u u}}{\Delta}, \\
& B=2 \frac{\psi_{u} \phi_{u v}-\phi_{u} \psi_{u v}}{\Delta}+\frac{\psi_{v} \phi_{u u}-\phi_{v} \psi_{u u}}{\Delta}, \\
& C=2 \frac{\psi_{v} \phi_{u v}-\phi_{v} \psi_{u v}}{\Delta}+\frac{\psi_{u} \phi_{v v}-\phi_{u} \psi_{v v}}{\Delta}, \\
& D=\frac{\psi_{v} \phi_{v v}-\phi_{v} \psi_{v v}}{\Delta}, \\
& \Delta=\phi_{u} \psi_{v}-\psi_{u} \phi_{v} \neq 0 .
\end{aligned}
$$

This is, consequently, the general form of the differential equation of a linear family in any system of coordinates.

If now the coordinates are minimal, then the necessary and sufficient condition for $\mathcal{F}$ to be a velocity family is, by comparison of (3.4) with (2.13), $A=0, D=0$, that is,

$$
\psi_{u} \phi_{u u}-\phi_{u} \psi_{u u}=0, \quad \psi_{v} \phi_{v v}-\phi_{v} \psi_{v v}=0
$$

This gives

$$
\frac{\phi_{u u}}{\phi_{u}}=\frac{\psi_{u u}}{\psi_{u}}=\rho, \quad \frac{\phi_{v v}}{\phi_{v}}=\frac{\psi_{v v}}{\psi_{v}}=\sigma
$$

or

$$
\rho=\left(\log \phi_{u}\right)_{u}=\left(\log \psi_{u}\right)_{u}, \quad \sigma=\left(\log \phi_{v}\right)_{v}=\left(\log \psi_{v}\right)_{v} .
$$

We calculate

$(\log \Delta)_{u}=\frac{\Delta_{u}}{\Delta}+\frac{\left(\phi_{u} \psi_{u v}-\psi_{u} \phi_{u v}\right)+\left(\phi_{u u} \psi_{v}-\psi_{u u} \phi_{v}\right)}{\Delta}=\frac{\phi_{u} \psi_{u v}-\psi_{u} \phi_{u v}}{\Delta}+\rho$, similarly

$$
(\log \Delta)_{v}=\frac{\Delta_{v}}{\Delta}=\frac{\psi_{v} \phi_{u v}-\phi_{v} \psi_{u v}}{\Delta}+\sigma
$$


so that the second and third equations of (3.5) can be written

$$
B=-2(\log \Delta)_{u}+3 \rho, \quad C=2(\log \Delta)_{v}-3 \sigma .
$$

The partial differential equations (3.6) are easily integrated, and the result may be written in the form

$$
U_{1} \phi+U_{2} \psi=1, \quad V_{1} \phi+V_{2} \psi=-1 ;
$$

therefore $\phi, \psi$ must have the forms

$$
\phi=\frac{U_{2}+V_{2}}{U_{1} V_{2}-U_{2} V_{1}}, \quad \psi=-\frac{U_{1}+V_{1}}{U_{1} V_{2}-U_{2} V_{1}},
$$

where $U_{1} V_{2}-U_{2} V_{1} \neq 0$; that is, $U_{1} / U_{2}$ and $V_{1} / V_{2}$ are not equal to the sa me constant, nor do we have $U_{1}=0$ and $U_{2}=0$ or $V_{1}=0$ and $V_{2}=0$.

From (3.11) we calculate

$$
\begin{aligned}
& \phi_{u}=V_{2} \frac{\left|\begin{array}{cc}
U_{1}+V_{1} & U_{1}^{\prime} \\
U_{2}+V_{2} & U_{2}^{\prime}
\end{array}\right|}{\left|\begin{array}{cc}
U_{1} & V_{1} \\
U_{2} & V_{2}
\end{array}\right|^{2}}, \quad \phi_{v}=-U_{2} \frac{\left|\begin{array}{cc}
U_{1}+V_{1} & V_{1}^{\prime} \\
U_{2}+V_{2} & V_{2}^{\prime}
\end{array}\right|}{\left|\begin{array}{cc}
U_{1} & V_{1} \\
U_{2} & V_{2}
\end{array}\right|^{2}}, \\
& \psi_{u}=-V_{1} \frac{\left|\begin{array}{cc}
U_{1}+V_{1} & U_{1}^{\prime} \\
U_{2}+V_{2} & U_{2}^{\prime}
\end{array}\right|}{\left|\begin{array}{cc}
U_{1} & V_{1} \\
U_{2} & V_{2}
\end{array}\right|^{2}}, \quad \psi_{v}=U_{1} \frac{\left|\begin{array}{cc}
U_{1}+V_{1} & V_{1}^{\prime} \\
U_{2}+V_{2} & V_{2}^{\prime}
\end{array}\right|}{\left|\begin{array}{cc}
U_{1} & V_{1} \\
U_{2} & V_{2}
\end{array}\right|^{2}},
\end{aligned}
$$

therefore, by the last equation of (3.5),

$$
\Delta=\frac{\left|\begin{array}{cc}
U_{1}+V_{1} & U_{1}^{\prime} \\
U_{2}+V_{2} & U_{2}^{\prime}
\end{array}\right| \cdot\left|\begin{array}{cc}
U_{1}+V_{1} & V_{1}^{\prime} \\
U_{2}+V_{2} & V_{2}^{\prime}
\end{array}\right|}{\left|\begin{array}{cc}
U_{1} & V_{1} \\
U_{2} & V_{2}
\end{array}\right|^{3}}
$$

By substitution of (3.12) in (3.8),

$$
\begin{aligned}
& \rho=\frac{\partial}{\partial u} \log \left|\begin{array}{cc}
U_{1}+V_{1} & U_{1}^{\prime} \\
U_{2}+V_{2} & U_{2}^{\prime}
\end{array}\right|-2 \frac{\partial}{\partial u} \log \left|\begin{array}{cc}
U_{1} & V_{1} \\
U_{2} & V_{2}
\end{array}\right|, \\
& \sigma=\frac{\partial}{\partial v} \log \left|\begin{array}{ll}
U_{1}+V_{1} & V_{1}^{\prime} \\
U_{2}+V_{2} & V_{2}^{\prime}
\end{array}\right|-2 \frac{\partial}{\partial v} \log \left|\begin{array}{cc}
U_{1} & V_{1} \\
U_{2} & V_{2}
\end{array}\right| .
\end{aligned}
$$


Substituting (3.13), (3.14) in (3.9), we find

$$
\begin{aligned}
& B=\frac{\partial}{\partial u} \log \left|\begin{array}{cc}
U_{1}+V_{1} & U_{1}^{\prime} \\
U_{2}+V_{2} & U_{2}^{\prime}
\end{array}\right|-2 \frac{\partial}{\partial u} \log \left|\begin{array}{cc}
U_{1}+V_{1} & V_{1}^{\prime} \\
U_{2}+V_{2} & V_{2}^{\prime}
\end{array}\right|, \\
& C=2 \frac{\partial}{\partial v} \log \left|\begin{array}{cc}
U_{1}+V_{1} & U_{1}^{\prime} \\
U_{2}+V_{2} & U_{2}^{\prime}
\end{array}\right|-\frac{\partial}{\partial v} \log \left|\begin{array}{cc}
U_{1}+V_{1} & V_{1}^{\prime} \\
U_{2}+V_{2} & V_{2}^{\prime}
\end{array}\right|,
\end{aligned}
$$

that is, as abbreviated by the notation (1.5),

$$
B=\frac{\partial}{\partial u}(\log \mathrm{I}-2 \log \mathrm{II}), \quad C=\frac{\partial}{\partial v}(2 \log \mathrm{I}-\log \mathrm{II}) .
$$

We thus have the result:

In order that a velocity family expressed in minimal coordinates be linear, it is necessary and sufficient that the coefficients $B, C$ in (2.13) have the special form (3.15) or (3.16).

To complete the imposition of property (3), in addition to the property (2) of linearity, we must particularize our velocity family by the additional condition (2.17): $C_{u}-B_{v}=2 k F$. Substituting from (3.16), this gives the result stated in formula (1.7):

$$
2 k F=\frac{\partial^{2}}{\partial u \partial v}(\log \mathrm{I}+\log \mathrm{II}) .
$$

We find by direct calculation :

$$
\begin{aligned}
\frac{\partial^{2}}{\partial u \partial v} \log I= & \frac{\left|\begin{array}{cc}
U_{1}+V_{1} & U_{1}^{\prime} \\
U_{2}+V_{2} & U_{2}^{\prime}
\end{array}\right| \cdot\left|\begin{array}{cc}
V_{1}^{\prime} & U_{1}^{\prime \prime} \\
V_{2}^{\prime} & U_{2}^{\prime \prime}
\end{array}\right|}{\left|\begin{array}{cc}
U_{1}+V_{1} & U_{1}^{\prime} \\
U_{2}+V_{2} & U_{2}^{\prime}
\end{array}\right|^{2}} \\
& +\frac{\left|\begin{array}{cc}
U_{1}+V_{1} & U_{1}^{\prime \prime} \\
U_{2}+V_{2} & U_{2}^{\prime \prime}
\end{array}\right| \cdot\left|\begin{array}{cc}
U_{1}^{\prime} & V_{1}^{\prime} \\
U_{2}^{\prime} & V_{2}^{\prime}
\end{array}\right|}{\left|\begin{array}{ll}
U_{1}+V_{1} & U_{1}^{\prime} \\
U_{2}+V_{2} & U_{2}^{\prime}
\end{array}\right|^{2}}
\end{aligned}
$$

The determinants which appear in the numerators are among the six in the matrix

$$
\left\|\begin{array}{cccc}
U_{1}+V_{1} & U_{1}^{\prime} & V_{1}^{\prime} & U_{1}^{\prime \prime} \\
U_{2}+V_{2} & U_{2}^{\prime} & V_{2}^{\prime} & U_{2}^{\prime}
\end{array}\right\|,
$$

which obey the well known identity $\left({ }^{13}\right)$ :

(18) The same as the one which governs Plücker line coordinates, being obeyed by the six determinants of second order in any two-by-four matrix. 


$$
\begin{aligned}
\left|\begin{array}{cc}
U_{1}+V_{1} & U_{1}^{\prime} \\
U_{2}+V_{2} & U_{2}^{\prime}
\end{array}\right| \cdot\left|\begin{array}{cc}
V_{1}^{\prime} & U_{1}^{\prime \prime} \\
V_{2}^{\prime} & U_{2}^{\prime \prime}
\end{array}\right|+ & \left|\begin{array}{cc}
U_{1}+V_{1} & U_{1}^{\prime \prime} \\
U_{2}+V_{2} & U_{2}^{\prime \prime}
\end{array}\right| \cdot\left|\begin{array}{cc}
U_{1}^{\prime} & V_{1}^{\prime} \\
U_{2}^{\prime} & V_{2}^{\prime}
\end{array}\right| \\
& =\left|\begin{array}{cc}
U_{1}+V_{1} & V_{1}^{\prime} \\
U_{2}+V_{2} & V_{2}^{\prime}
\end{array}\right| \cdot\left|\begin{array}{cc}
U_{1}^{\prime} & U_{1}^{\prime \prime} \\
U_{2}^{\prime} & U_{2}^{\prime \prime}
\end{array}\right| .
\end{aligned}
$$

Therefore

$$
\frac{\partial^{2}}{\partial u \partial v} \log \mathrm{I}=\frac{\mathrm{II}}{\mathrm{I}^{2}} \cdot\left|\begin{array}{cc}
U_{1}^{\prime} & U_{1}^{\prime \prime} \\
U_{2}^{\prime} & U_{2}^{\prime \prime}
\end{array}\right| .
$$

Similarly we find

$$
\frac{\partial^{2}}{\partial u \partial v} \log \mathrm{II}=\frac{\mathrm{I}}{\mathrm{II}^{2}} \cdot\left|\begin{array}{ll}
V_{1}^{\prime} & V_{1}^{\prime \prime} \\
V_{2}^{\prime} & V_{2}^{\prime \prime}
\end{array}\right| .
$$

These are the formulas stated as (1.8); substituted in (3.17), they give the expanded form $\left(1.7^{\prime}\right)$ for $2 k F$.

The proof of our main results is thus completed.

4. General coordinates. It is interesting to see how our formulas look in general coordinates $u, v$, instead of minimal coordinates.

Using the general formula $\left({ }^{14}\right)$ for geodesic curvature $1 / \rho$, the condition (2.10) for a velocity family gives the following characteristic differential equation for such a family:

$$
\begin{aligned}
W^{2} v^{\prime \prime}= & W\left(P+Q v^{\prime}\right)\left(E+2 F v^{\prime}+G v^{\prime 2}\right) \\
& +\left(F+G v^{\prime}\right)\left[\frac{1}{2} E_{u}+E_{v} v^{\prime}+\left(F_{v}-\frac{1}{2} G_{u}\right) v^{\prime 2}\right] \\
& -\left(E+F v^{\prime}\right)\left[\left(F_{u}-\frac{1}{2} E_{v}\right)+G_{u} v^{\prime}+\frac{1}{2} G_{v} v^{\prime 2}\right] .
\end{aligned}
$$

The imposition of property (3) is completed by (2.11), where $K$ is to be thought of as expressed in terms of $E, F, G$ and their first and second partial derivatives $\left({ }^{15}\right)$ :

$$
Q_{u}-P_{v}=(k-K) W .
$$

We have also to express property (2), of linearity, in general coordinates. It is a known result $\left({ }^{16}\right)$ that the most general linear family has the form

$$
v^{\prime \prime}=A+B v^{\prime}+C v^{\prime 2}+D v^{\prime 3},
$$

where $A, B, C, D$ are functions of $u, v$ which obey the conditions

$$
\begin{aligned}
\left(A C-A_{v}\right)_{v}-(A D+ & \left.\frac{1}{3} C_{u}-\frac{2}{3} B_{v}\right)_{u} \\
& +B\left(A D+\frac{1}{3} C_{u}-\frac{2}{3} B_{v}\right)-A\left(B D+D_{u}\right)=0,
\end{aligned}
$$

\footnotetext{
(14) Blaschke, loc. cit., p. 117.

(16) See formula (4.11).

(16) Due to Lie and R. Liouville. See E. Kasner [1].
} 


$$
\begin{aligned}
-\left(B D+D_{u}\right)_{u}+(A D+ & \left.\frac{2}{3} C_{u}-\frac{1}{3} B_{v}\right)_{v} \\
& +C\left(A D+\frac{2}{3} C_{u}-\frac{1}{3} B_{v}\right)-D\left(A C-A_{v}\right)=0 .
\end{aligned}
$$

The formula (4.1) is of the type (4.3) with

$$
\begin{aligned}
& A=\left\{W P E+\frac{1}{2} F E_{u}-E\left(F_{u}-\frac{1}{2} E_{v}\right)\right\} / W^{2}, \\
& B=\left\{2 W P F+W Q E+F E_{v}+\frac{1}{2} G E_{u}-F\left(F_{u}-\frac{1}{2} E_{v}\right)-E G_{u}\right\} / W^{2}, \\
& C=\left\{W P G+2 W Q F+G E_{v}+F\left(F_{v}-\frac{1}{2} G_{u}\right)-\frac{1}{2} E G_{v}-F G_{u}\right\} / W^{2}, \\
& D=\left\{W Q G+G\left(F_{v}-\frac{1}{2} G_{u}\right)-\frac{1}{2} F G_{v}\right\} / W^{2} .
\end{aligned}
$$

The conditions (4.4), (4.5) of linearity give two partial differential equations involving $E, F, G, P, Q$.

In summary: configurations $(\mathcal{F}, S)$ having properties (2) and (3) are characterized in general coordinates by

$$
\begin{aligned}
& S: \quad d s^{2}=E d u^{2}+2 F d u d v+d v G^{2}, \\
& \mathcal{F}: \quad \text { of type (4.1), }
\end{aligned}
$$

where the five functions $E, F, G, P, Q$ obey the three partial differential equations (4.2), (4.4), (4.5), in which $A, B, C, D$ are defined by (4.6). These equations are of the third order in $E, F, G$ and the second order in $P, Q$.

Of course, the general solution is obtainable by applying an arbitrary transformation $u_{1}=\phi(u, v), v_{1}=\psi(u, v)$ to the formulas based on minimal coordinates.

We may inquire also as to the form our equations have in the particular coordinate system $(u, v)$ where the equations of $\mathcal{F}$ are linear $\left({ }^{17}\right): v=a u+b$, or $v^{\prime \prime}=0$. In (4.6), we have then to write

$$
A=0, \quad B=0, \quad C=0, \quad D=0,
$$

which, in addition to (4.2), give a system of five conditions on $E, F, G, P, Q$. From these we easily eliminate $P, Q$ and obtain the following three conditions on $E, F, G$ :

$$
\begin{aligned}
& \left(\frac{1}{2} E G-F^{2}\right) \frac{E_{u}}{E}+\frac{1}{2} F E_{v}+F F_{u}-E F_{v}-\frac{1}{2} E G_{u}+\frac{1}{2} E F \frac{G_{v}}{G}=0, \\
& \left(\frac{1}{2} E G-F^{2}\right) \frac{G_{v}}{G}+\frac{1}{2} F G_{u}+F F_{v}-G F_{u}-\frac{1}{2} G E_{v}+\frac{1}{2} F G \frac{E_{u}}{E}=0, \\
& 2 W^{3} \frac{\partial}{\partial v} \frac{F E_{u}-E F_{u}}{W E}+2 W^{3} \frac{\partial}{\partial u} \frac{F G_{v}-G F_{v}}{W G}-\left|\begin{array}{ccc}
E & E_{u} & E_{v} \\
F & F_{u} & F_{v} \\
G & G_{u} & G_{v}
\end{array}\right|=4 k W^{4} .
\end{aligned}
$$

${ }^{(17)}$ Of course, this coordinate system is determined only up to an arbitrary projective transformation of $u, v$. 
In the derivation of (4.10), we use the formula of G. Frobenius for Gaussian curvature $\left({ }^{18}\right)$ :

$$
K=-\frac{1}{4 W^{4}}\left|\begin{array}{lll}
E & E_{u} & E_{v} \\
F & F_{u} & F_{v} \\
G & G_{u} & G_{v}
\end{array}\right|-\frac{1}{2 W}\left\{\frac{\partial}{\partial v} \frac{E_{v}-F_{u}}{W}-\frac{\partial}{\partial u} \frac{F_{v}-G_{u}}{W}\right\} .
$$

In summary, (4.8)-(4.10) are necessary and sufficient conditions on the $E, F, G$ of a surface in order that the curve family defined by the general linear equation, $v=a u+b$, have the property $\varepsilon=k \mathcal{A}$.

(4.8) and (4.9) are necessary and sufficient in order that $v=a u+b$ shall be a velocity family.

5. Geometric construction for the property $\varepsilon=k \mathcal{A}$. In the case $k=0$, it is well known that the curve families $\mathcal{F}$ which have the property: $\mathcal{E}=0$ or $A+B+C=\pi$ for every triangle $A B C$ of $\mathcal{F}$, are exactly the $i$ sogonal families $\left({ }^{19}\right)$. These consist of the totality of $\infty^{2}$ trajectories under every possible constant angle $\theta$ of any given family $\alpha$ of $\infty^{1}$ curves.

It is easy to generalize this construction to the case $k \neq 0$. Take any net of curves upon an arbitrary surface $S$, composed of two families $\alpha, \beta$ of $\infty^{1}$ curves. Construct a trajectory $T$ of the family $\alpha$, not under constant angle, but rather so that the angle $\theta$ between $T$ and $\alpha$ decreases by $k$ times the element of area swept out by the arc of the curve $\beta_{p}$ of $\beta$ which passes through the point $p$ describing $T$ and extends to the intersection $m$ of $\beta_{p}$ with any chosen fixed curve $\alpha_{0}$ of $\alpha$.

That is-with reference to a figure easily drawn by the reader-we have, in integrated form, the law

$$
\theta_{2}-\theta_{1}=-k \cdot \text { area } p_{1} p_{2} m_{1} m_{2}
$$

for the construction of $T$. Evidently, this law determines the formation of $T$, element by element, when any initial point and direction are given; therefore the totality of trajectories $T$ is a family $\mathcal{F}$ of $\infty^{2}$ curves. It is very easy to give by means of a figure a proof of the property: $\mathcal{E}=k \mathcal{A}$ for every triangle of $\mathcal{F}$.

It remains to be shown that every family $\mathcal{F}$ with the property $\mathcal{E}=k \mathcal{A}$ is obtainable by a construction of the type just described. This is readily done by the following converse reasoning. Construct the pencil $\Pi$ of curves of $\mathcal{F}$ through a fixed point $p$. In the region $R$ covered by $\Pi$, construct any family $\beta$ of $\infty^{1}$ curves all of which intersect a fixed base curve $\alpha_{0}$. At each point of $R$ construct a direction $\delta$ according to the following law: (i) at the points of $\alpha_{0}$, $\delta$ shall coincide with the tangential direction to $\alpha_{0}$; (ii) the angle $\theta$ between $\Pi$ and $\delta$ shall vary according to the law (5.1). We thus have a field of directions

(18) Blaschke, loc. cit., p. 79.

(19) G.Scheffers, Isogonalkurven, Äquitangentialkurven und komplexe Zahlen, Mathematische Annalen, vol. 60 (1905), p. 504. 
$\delta$, whose integration gives a family of $\infty^{1}$ curves $\alpha$, including $\alpha_{0}$. If now $T$ is any curve of the family $\mathcal{F}$ lying in the region $R$, it is evident, by applying the property $\mathcal{E}=k \mathcal{A} A$ to the triangle formed by any arc $p_{1} p_{2}$ of $T$ and the curves $p p_{1}, p p_{2}$ of $\Pi$, that $T$ traverses $\alpha$ according to the law (5.1).

Thus the law (5.1) holds as long as $T$ lies in the region $R$ covered by $\Pi$. We can extend this region by applying the same reasoning to the pencil of curves of $\mathcal{F}$ which pass through any other fixed point $p^{\prime}$ of $R$, and repeating this procedure any finite number of times.

6 . Higher dimensions, $n>2$. We conclude with a statement of the analogous problem for higher dimensional spaces, which we hope to consider in a future paper.

Let $\mathcal{F}$ denote a linear family of curves in a space of $n>2$ dimensions; that is, let $\mathcal{F}$ be depictable as the totality of straight lines of a flat projective $n$-space $P$. It is required to impose on the space $P$ a Riemannian metric $R: d s^{2}=g_{i j} d x^{i} d x^{i}$, so that, in every triangle of $P, \mathcal{E}=k \mathcal{A}$ with $k$ a preassigned nonzero constant, angles and areas being measured according to $R$.

Certainly, a sufficient condition is that the space $R$ have constant Riemannian curvature and that $\mathcal{F}$ consist of its geodesics. In other words, $R$ shall be the Cayley metric based on any fixed quadric $Q$ : dist $a b=\left\{2(-k)^{1 / 2}\right\}^{-1}$ $\cdot \log \left(a b p_{1} p_{2}\right)$, where $p_{1}, p_{2}$ are the intersection points of the line $a b$ with $Q$, and the parenthesis denotes an anharmonic ratio. For the Cayley metric is a typical one of constant curvature, and the straight lines are its geodesics.

It remains to be seen whether, for $n>2$, the Cayley metric is the most general one which can be imposed on the projective space $P$ so that $\varepsilon=k \mathcal{A}$. We reserve this problem for future consideration.

It may be remarked that for $k=0$ and $n>2$ the property $\varepsilon=0$ - that is, the property that the angle-sum of every triangle of $\mathcal{F}$ is two right anglesimplies that $\mathcal{F}$ is linear $\left({ }^{20}\right)$. The author has proved that, furthermore, it must be possible to represent the Riemann space $R$ conformally on a euclidean space $E$ so that $\mathcal{F}$ corresponds to the straight lines of $E\left({ }^{20}\right)$.

\section{REFERENCES}

1. E. Kasner, A characteristic property of isothermal systems of curves, Mathematische Annalen, vol. 59 (1904), pp. 352-354.

2. J. Douglas, $A$ criterion for the conformal equivalence of a Riemann space to a euclidean space, these Transactions, vol. 27 (1925), pp. 299-306.

3. J. Radon, Kurvennetze auf Flächen und im Raume von Riemann, Abhandlungen aus dem mathematischen Seminar der Hamburgischen Universität, vol. 5 (1927), pp. 45-53.

4. C. H. Rowe, On certain systems of curves in Riemannian space, Journal de Mathématiques Pures et Appliquées, vol. 12 (1933), pp. 283-308.

BROOKLYN, N. Y.

(20) Reference [2]. 